\title{
CAPACIDAD DE AGENCIA DE AUTOCUIDADO EN LAS PERSONAS ADULTAS QUE PADECEN ARTRITIS REUMATOIDE ${ }^{1}$
}

\author{
Viriam Leiva Díaz ${ }^{2}$ \\ Pilar Acosta Rojas ${ }^{3}$ \\ Yara Berrocal Barboza ${ }^{4}$ \\ Erica Carrillo Sancho ${ }^{5}$ \\ Marjorie Castro Marin ${ }^{6}$ \\ Yahaira Watson Guido ${ }^{7}$
}

Institución: Universidad de Costa Rica

COMO CITAR

Leiva, V.; Acosta, P.; Berrocal, Y.; Carrillo, E.; Castro, M.; Watson, Y.(2012).Capacidad de Agencia de Autocuidado en personas adultas con artritis reumatoide.[En línea].Rev. Enfermería Actual en Costa Rica, 22, 1-11 [citado (fecha)]. Disponible World Wide Web:

$<$ http://www.revenf.ucr.ac.cr/artritis.pdf> ISSN 1409-4568

\section{RESUMEN}

El objetivo de la investigación consistió en analizar la capacidad de agencia de autocuidado que poseen las personas con diagnóstico de artritis reumatoide, quienes habitan en los cantones de Palmares y San Ramón. Se desarrolló una metodología de investigación cuantitativa de tipo descriptiva transversal. En cuanto a la población participante del estudio, estuvo conformada por 13 personas diagnosticadas con artritis reumatoide; respecto de los instrumentos utilizados para recolectar información, a los colaboradores se les aplicó el instrumento "Escala de apreciación de la capacidad de agencia de autocuidado", propuesta por Dorothea Orem. Se determinó que los participantes poseen alta capacidad de agencia de autocuidado en las diferentes categorías, ya que únicamente se detectó baja capacidad de agencia de autocuidado en cuanto a interacción social (2 personas), y respecto del consumo insuficiente de alimentos (5 personas). La escala ASA permite a la Enfermera (o) determinar la capacidad de agencia de autocuidado, de forma oportuna y eficaz. Además, permitió a las investigadoras observar un panorama general de las necesidades de autocuidado de la población participante y constatar que esta presenta alta capacidad de agencia de autocuidado.

Palabras clave: autocuidado, Dorothea-Orem, artritis-reumatoide, capacidad-de-agencia-de-autocuidado

${ }^{1}$ Fecha de recepción: 26 de julio del 2011

Fecha de aceptación: 15 de enero del 2012

${ }^{2}$ Viriam Leiva Díaz. Profesora catedrática de la Escuela de Enfermería de la Universidad de Costa Rica. Enfermera. Psicóloga. Magister en Psicopedagogía. Investigadora del Programa de Investigación en Enfermería y del Instituto de Investigaciones Filosóficas de la Universidad de Costa Rica. Correo electrónico: viriaml@gmail.com

${ }^{3}$ Pilar Acosta Rojas. Licenciada en Enfermería, trabaja en el Hospital Cima. Correo electrónico: pilyacos7@hotmail.com

${ }^{4}$ Yara Berrocal Barboza.Licenciada en Enfermería, trabaja en el Hospital Cima. Correo electrónico: tinygirl15@hotmail.com

${ }^{5}$ Maryorie Castro Marín: Licenciada en Enfermería, trabaja en el Hospital Cima. Correo electrónico: marjoriemcm@gmail.com

${ }^{6}$ Erica Carrillo Sancho. Licenciada en Enfermería, trabaja en el Hospital Hotel la Católica. Correo electrónico: erica2708c@gmail.com

${ }^{7}$ Yahaira Watson Guido. Licenciada en enfermería. Labora propio. Correo electrónico: yajawatson@hotmail.com 


\section{ABILITY TO SELF-CARE AGENCY IN ADULTS WITH RHEUMATOID ARTHRITIS ${ }^{1}$}

Institution: Universidad de Costa Rica

Viriam Leiva Díaz ${ }^{2}$

Pilar Acosta Rojas ${ }^{3}$

Yara Berrocal Barboza ${ }^{4}$

Erica Carrillo Sancho 5

Marjorie Castro Marin ${ }^{6}$

Yahaira Watson Guido ${ }^{7}$

CITED

Leiva, V.; Acosta, P.; Berrocal, Y.; Carrillo, E.; Castro, M.; Watson, Y. (2012). Ability to self-care agency in adults with rheumatoid arthritis.[Electronic version].Rev. Enfermería Actual en Costa Rica, 22, 1-11 [cited (date)]. Available World Wide Web: <http://www.revenf.ucr.ac.cr/artritis.pdf> ISSN $1409-4568$

\section{ABSTRACT}

The objective of this research was to analyze the capacity of self-care agency that people have been diagnosed with rheumatoid arthritis, in the cantons of Palmares and San Ramon. This was achieved through a quantitative research methodology, descriptive transversal study. Participant population was 13 people diagnosed with rheumatoid arthritis; the collaborators were administered the instrument "Scale Assessment of Self-Care Agency Capacity" proposed by Dorothea Orem. As results of the study found that participants have a high capacity selfcare agency in the different categories, only in terms of social interaction, 2 persons, and sufficient intake of food, 5 people got low self-care agency capacity. The ASA scale allows the Nurse (o) determine the ability of self-care agency, in a timely and effective. It also allowed the researchers see an overview of self-care needs of the participant population and find that present high capacity self-care agency.

Keywords: self-care, rheumatoid-arthritis, Dorothea-Orem, ability-to-self-care-agency

\footnotetext{
${ }^{1}$ Date of reception: July 26, 2011

${ }^{2}$ Viriam Leiva Díaz. Profesora catedrática de la Escuela de Enfermería de la Universidad de Costa Rica. Enfermera. Psicóloga. Magister en Psicopedagogía. Investigadora del Programa de Investigación en Enfermería y del Instituto de Investigaciones Filosóficas de la Universidad de Costa Rica. E. mail: viriaml@gmail.com

${ }^{3}$ Pilar Acosta Rojas. Licenciada en Enfermería, trabaja en el Hospital Cima. E. mail: pilyacos7@hotmail.com

${ }^{4}$ Yara Berrocal Barboza.Licenciada en Enfermería, trabaja en el Hospital Cima. E. mail: tinygirl15@hotmail.com

${ }^{5}$ Maryorie Castro Marín: Licenciada en Enfermería, trabaja en el Hospital Cima. E. mail: marjoriemcm@gmail.com

${ }^{6}$ Erica Carrillo Sancho. Licenciada en Enfermería, trabaja en el Hospital Hotel la Católica. E. mail: erica2708c@gmail.com

${ }^{7}$ Yahaira Watson Guido. Licenciada en enfermería. Labora propio. E. mail: yajawatson@ hotmail.com
}

Date of acceptance: January 15, 2012 


\section{INTRODUCCIÓN}

La disfunción del sistema formado por huesos y articulaciones es una anomalía frecuente en la clínica y también una de las causas más comunes de consulta en la Atención Primaria en nuestro país. Asimismo, se sabe que entre el 10\% y el $40 \%$ de la población general padece algún trastorno osteoarticular, situación que muestra cuán importante es realizar estudios relacionados con tales patologías, principalmente si el objetivo es beneficiar o facilitar la atención de las personas que lo padecen.

En Costa Rica no se conocen datos estadísticos sobre la cantidad de personas con artritis reumatoide (Arias, 2010); sin embargo, el comportamiento de la enfermedad es similar a nivel mundial, por lo que se estima que sea equivalente al 1\% del total de habitantes; a pesar de que la cantidad de individuos con la patología es considerable, dicha población ha sido poco estudiada en el ámbito nacional y su atención, en la mayoría de los casos, se centra en la consulta con el médico reumatólogo, dejando de lado las distintas esferas del ser humano.

Respecto de lo anterior, debido a las características de la enfermedad, la mitad de las personas diagnosticadas experimentan, aun después de cinco años de habérseles descubierto el padecimiento, dificultades para mantener un nivel satisfactorio en cuanto a actividad física y desempeño laboral, ámbito que se ve afectado luego de diez o quince años posteriores al diagnóstico, máxime que durante la mitad de este periodo los pacientes presentan dificultad para ejercer alguna actividad laboral y dos tercios se enfrentan con problemas en las actividades de la vida diaria. Por las razones mencionadas, dentro de la atención es imperante determinar cuántas personas poseen un trabajo estable, así como en qué áreas de la vida se detectan problemas, para de este modo brindar una atención oportuna y eficaz que se base en las características propias de cada individuo.

La importancia de conocer estas implicaciones desde el punto de vista de enfermería radica en las características propias de la enfermedad pueden comprometer la capacidad de la persona para realizar su autocuidado. La limitación para desarrollar los roles y actividades en un ambiente definido es una de las principales consecuencias, ya que puede afectar el ámbito personal, laboral y social y dificultar la forma en la que se maneja la enfermedad.

Por otro lado, Orem (1983) menciona que el profesional en enfermería debe ser capaz de identificar las necesidades de las personas que atiende, seleccionar el o los métodos generales de ayuda que requiere el individuo para que sus requisitos de autocuidado o necesidades sean cubiertas, así como identificar las acciones específicas para lograr el objetivo y resultado deseado. Es por ello que en la presente investigación se trabajó con la Teoría General de Enfermería (Orem, 1983), sido utilizada en diversos lugares del mundo (Colombia, Estados Unidos, Hong Kong, entre otros) (Fernández, 2010). Su uso se ha expandido al punto de que se ha visto enriquecida por distintos aportes entre los cuales debe mencionarse la Escala de Apreciación de Agencia de Autocuidado (ASA), creada por Evers, Isenberg, Philipsen, Senten, Brouns(1993); dicha escala presenta ventajas para su utilización, por ejemplo, cuenta con la facilidad de ser autoadministrada o dirigida y, al presentar solamente 24 ítems, requiere un periodo corto para su aplicación (entre 10 y 20 minutos), por lo que mantiene el interés de la persona que la está completando, de manera que se evita sesgos de información confiable.

Asimismo, al utilizar el ASA, el déficit en la capacidad de autocuidado se puede detectar de forma oportuna y adecuada, de modo que se brinda una atención más específica según las necesidades de cada uno de 
los grupos clasificados, mejora que reduce tanto la inversión económica directa (hospitalizaciones-consultas y tratamiento) como la indirecta por parte de las instituciones de salud; además, se agilizaría la atención y se enfocaría en las áreas que obtengan puntajes inferiores, de forma que contribuya con el bienestar y funcionamiento personal.

Es por ello que a pesar de que los resultados de esta investigación no se generalizan, pueden ser aplicables a grupos con similares características y establecer pautas para la identificación temprana y oportuna de problemas en tales pacientes, así como implementación de medidas necesarias para su abordaje. Por ello, el análisis del tema es de interés y beneficio quienes la padecen, debido a que los sistemas de salud deberían considerar las fortalezas, limitaciones y necesidades detectadas en esta investigación lo que permite proporcionar alternativas como la creación de programas destinados a educar y capacitar a la persona con diagnóstico de la artritis reumatoide y que propicien una atención de calidad.

\section{MATERIALES Y MÉTODOS}

La investigación fue cuantitativa de tipo descriptiva transversal, puesto que los resultados se expresaron en números enteros, además de que el fin de esta fue expresar propiedades de las personas, analizando cada una de las variables de forma independiente, pues no se pretendía indicar cómo se correlacionan. (Barrantes, 2002)

La población participante estuvo compuesta por 13 personas con diagnóstico de artritis reumatoide (10 mujeres y 3 hombres) por lo que al ser una población pequeña, no se realizó muestreo. Estas personas fueron contactadas por medios externos a los entes públicos de salud, debido a que estos no permitieron el acceso a información. Como criterios de inclusión se buscó individuos que vivieran en los cantones de Palmares y San Ramón, mayores de 18 años de edad, con un diagnóstico posterior a un año y que tuvieran disposición para participar en el estudio. Se excluyó aquellos con trastornos de la capacidad mental, con limitaciones en la comunicación verbal o que no cumplieran con los requisitos antes mencionados, puesto que las investigadoras no contaban con la preparación para atender este tipo de población.

A los participantes les fue aplicado el instrumento "Escala de Apreciación de la Capacidad de Agencia de Autocuidado" (Gallegos, 1998; Fernández, Manrique, Velandia, 2009). Dicho instrumento cuenta con 24 ítems, con cuatro alternativas de respuesta, y con el cual se evalúa la capacidad de agencia de autocuidado en las categorías de interacción social, consumo suficiente de alimentos, bienestar personal, promoción del funcionamiento y desarrollo personal y actividad y reposo.

Para la realización de este estudio se tomaron en cuenta todas las consideraciones éticas respectivas. El Consejo de Organizaciones Internacionales de las Ciencias Médicas (CIOMS) afirma que la investigación se puede justificar desde el punto de vista ético sólo si se respeta y protege a los sujetos que participan de la investigación y sólo si lo realizado es moralmente aceptable en la comunidad (CIOMS, 2002). Por lo tanto, se incluyeron los siguientes aspectos:

-La autonomía (García, 2005): se dio libertad a las y los participantes de expresar lo deseado.

-La privacidad: se mantuvo el anonimato de las y los participantes: en ningún momento se revelaron sus nombres u otros detalles no pertinentes a la investigación. 
-El consentimiento informado: fue impreso y dado a cada una de las y los participantes. Este constó de información detallada sobre la investigación, su propósito, lo que se pretendía realizar, los riesgos y beneficios, además de los datos precisos sobre las investigadoras. Fue firmado por voluntad propia por las y los sujetos de investigación posterior a su lectura, explicación detallada y la aclaración de las consultas que surgieron en el momento.

-Divulgación de resultados: se realizó por medio del presente artículo científico.

Cabe aclarar que este aspecto fue contemplado en el consentimiento informado.

\section{RESULTADOS}

Respecto del lugar de procedencia, los datos mostraron que siete de los participantes son residentes de San Ramón, mientras que los otros 6 son procedentes de Palmares. En cuanto al sexo, de los 13 entrevistados, 10 son del género femenino, mientras que 3 pertenecían al masculino. La edad promedio de los individuos participantes en el estudio fue de 60 años: la mujer más joven es una señora que se encuentra entre las edades de 40-50 y la mayor con más de 60 años; en cuanto a los hombres, las edades se encontraron en los rangos de 40 y más de 60 años. Por otra parte, en cuanto al estado civil de los participantes, 10 de las personas son casadas, una manifestó estar viuda y dos, divorciados. Asimismo, en relación con el número de hijos, tres individuos tienen entre 1-2 hijos; cuatro, entre 3-4 hijos; cinco, más de 4 y solamente una persona mencionó no tener hijos.

Por otra parte, en cuanto a la escolaridad de los entrevistados, 3 personas tienen estudios primarios incompletos; 6 individuos, primaria completa; 1 persona cuenta con secundaria incompleta y tres personas poseen estudios universitarios. De igual manera, el total de la población cuenta con un Sistema de Seguridad Social.

El gráfico 1 muestra los resultados generales obtenidos de la escala ASA, los cuales demuestran que la población participante posee alta Capacidad de Agencia de Autocuidado:

\section{Gráfico 1}

\section{Palmares, San Ramón: Capacidad de Agencia de Autocuidado según} Categorías propuestas por Orem, 2010.

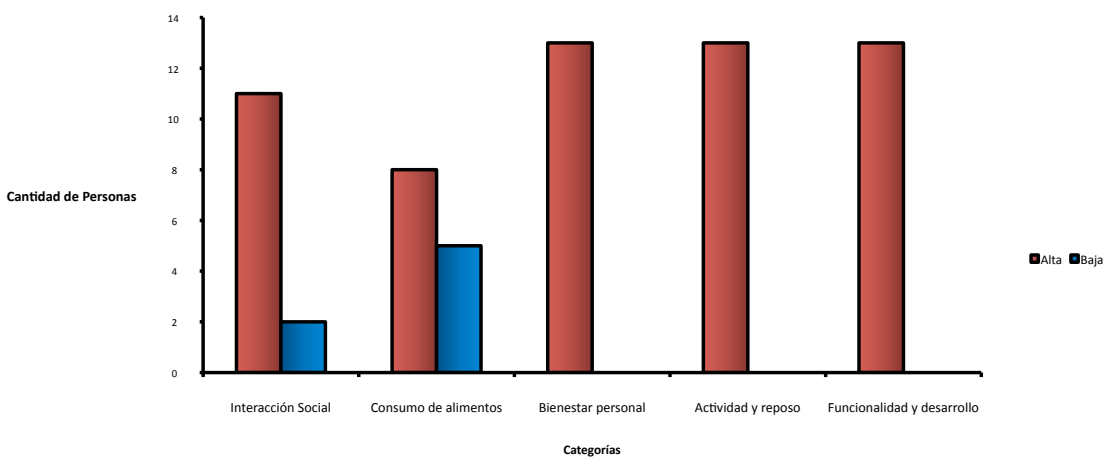


En cuanto a la categoría de interacción social, se demostró que 11 de los participantes poseen alta capacidad de agencia de autocuidado, mientras que 2 de las personas están dentro del parámetro de baja capacidad de autocuidado. En relación con la categoría de consumo suficiente de alimentos, 5 personas poseen baja Capacidad de Agencia de Autocuidado. Finalmente, en las categorías de bienestar personal, actividad y reposo, así como funcionamiento y desarrollo personal, el total de la población se ubicó en el parámetro Alta Capacidad de Agencia de Autocuidado.

\section{DISCUSIÓN}

El estudio pretendió identificar la capacidad de Agencia de Autocuidado de un grupo de personas con diagnóstico de artritis reumatoide, quienes son vecinos de los cantones de Palmares y San Ramón, pertenecientes a la provincia de Alajuela, Costa Rica. Los resultados encontrados demostraron que, según lo propuesto por Orem (1983), la salud se encuentra estrechamente unida a la cultura de la comunidad, por tal motivo, el profesional de enfermería debe identificar las características del lugar y, a partir de ello, conocer aquellos factores de protección o de riesgo que rodean el ambiente del individuo.

Los datos confirman la importancia de las variables sociodemográficas en la capacidad de Agencia de Autocuidado, pues en relación con el género, se evidencia que las mujeres tienen tres veces más probabilidad que los hombres de desarrollar artritis reumatoide, debido a los roles establecidos para el género femenino, pues son las mujeres quienes, por realizar más cantidad de actividades manuales, son más propensas a sufrir problemas interarticulares. Lo anterior evidencia que el género influye en la capacidad de Agencia de Autocuidado, así como en la realización del autocuidado. (Álvarez, 2003)

Asimismo, el género también influye como un factor protector o agresor en la capacidad de Agencia de Autocuidado que realizan las personas. Al respecto Matud (2008) indica que son las mujeres quienes presentan más conductas protectoras de la salud, en comparación con los hombres, debido a que estos últimos recurren menos a centros de salud o lo hacen en periodos avanzados de la enfermedad, conducta que incrementa el deterioro e impide un adecuado proceso de atención, calidad de vida y bienestar.

Conocer la edad en la investigación permitió a las investigadoras valorarla como un índice de las dimensiones de salud, pues en cada etapa de la vida los individuos van a presentar necesidades que deben abordarse según las demandas de cada ciclo de vida. Esto, permite aclarar su capacidad de mantener y realizar su autocuidado, sin olvidar la influencia de factores hereditarios o ambientales.

La edad y el género se relacionan con la posición dentro de la familia, pues determinan las ocupaciones y responsabilidades de la persona en el hogar (Orem, 1983), por lo tanto, debido a la disminución de la movilidad que puede generar la enfermedad, es fundamental valorar los rubros mencionados, puesto que las relaciones e interacción social pueden verse afectadas y comprometer aún más la condición de salud.

Otro aspecto sociodemográfico analizado fue el estado civil, cuya importancia radica en que los familiares constituyen un agente de autocuidado dependiente que proporciona cuidado a quienes tienen un diagnóstico de artritis reumatoide, cuando estos no pueden hacerlo por sí mismos. Este apoyo brindado por los diferentes miembros de la familia desempeña una importante función en cuanto a sobrellevar las características propias de la 
enfermedad, pues, tal como lo demostró el estudio realizado por Anaya, Cadena, Tobón y Vinaccia (2004), el apoyo social de la red familiar es un predictivo para un buen manejo de la ansiedad y depresión.

Por tal motivo, el que una persona cuente con la ayuda de los diferentes miembros de la familia va a contribuir con un desarrollo adecuado del proceso de la enfermedad, creando un estado mental positivo ante la misma, así como un mejor funcionamiento y desarrollo humano. Según Álvarez, et al.(2002), la familia constituye un soporte emocional para las personas con artritis reumatoide, dado que con su apoyo promueven el bienestar psicológico y la calidad de vida, de tal forma que constituye un elemento que permite a los profesionales en la salud evaluar el impacto de la enfermedad y el tratamiento en las personas.

Se consultó sobre la escolaridad, pues de esta forma se puede conocer si la preparación académica de un individuo puede afectar la percepción de las necesidades y demandas de autocuidado e, incluso, la posibilidad real de acceso a bienes y servicios de salud. El bienestar intelectual y ocupacional permite a la persona satisfacer las necesidades básicas, en especial la de individuos que padecen una enfermedad crónica (Guisti, citado por Araya, Mora, Ozols y Villalobos, 2004); dichas herramientas facilitan el manejo de la patología y contribuyen a mantener un mejor estado psicoemocional, para enfrentar las diferentes etapas de la enfermedad.

Finalmente, se indagó sobre el acceso y uso de los servicios de salud; el total de las personas indicó que asiste a la Clínica de San Ramón, para el seguimiento y control farmacológico de la enfermedad, como factor protector; lo que evidencia que los participantes en el estudio cuentan con afiliación a la seguridad social, beneficio que facilita la regulación de los procesos de la enfermedad y rehabilitación, en caso de que la movilidad se vea limitada o aparezcan complicaciones de la patología; al respecto, Orem (1983) establece que el "individuo que está bajo cuidado sanitario, contará con un mantenimiento y promoción de la salud” (p. 219), es decir, la persona tendrá a su alcance la facilidad de regular los procesos de la enfermedad y rehabilitación para poder vivir y actuar con facilidad y satisfacción personal durante todo el proceso de su enfermedad.

Para enmarcar la discusión de los resultados de la Escala de Apreciación de Autocuidado (ASA), es necesario definir qué se entiende por Capacidad de Agencia de Autocuidado; para ello se utiliza la conceptualización dada por Orem (1983), quien la define como la capacidad que permite a las personas discernir los factores que deben ser controlados o tratados para regular su propio funcionamiento y desarrollo, de manera que cubran sus requisitos de autocuidado.

A continuación, se presenta el análisis de las categorías en las que se clasifica la Escala ASA; cada una de ellas corresponde a los requisitos de autocuidado planteados por Orem (1983), es decir, representan las acciones que originan las condiciones internas y externas que mantiene, el funcionamiento adecuado del ser humano, así como su desarrollo. Dado lo anterior, es esencial comprender cada una de dichas categorías, para que el profesional de enfermería pueda crear y proyectar el planeamiento de la atención, de acuerdo con las demandas que presente grupo por tratar.

Respecto de la categoría de interacción social, según Orem (1983), este es un requisito de autocuidado que requiere ser satisfecho para el control temprano de la patología. Según lo planteado, se requiere un equilibrio entre la soledad y la interacción social, puesto que el individuo es un ser sociable que forma parte de un conjunto de personas, desde su nacimiento hasta su muerte, el cual inicia con el grupo familiar. Así, lo describe Rigol y 
Ugalde (2001), al afirmar que la familia, la sociedad y la cultura, son elementos básicos para que la persona pueda satisfacer sus necesidades, pues a través de la interacción del sujeto con dichos elementos, este adquiere el aprendizaje, madurez y herramientas para realizar su autocuidado.

Es de relevancia destacar que los requisitos de autocuidado se encuentran relacionados entre sí. Desde ese punto, se puede destacar que la interacción social contribuye al mantenimiento de la integridad de la persona, así como en la promoción del funcionamiento y su desarrollo. Esto, se resumiría en bienestar personal que según Johnson, et al. (2007) es definido como el "alcance de la percepción positiva del estado de salud y de las circunstancias vitales propias"(p. 693)

A partir de lo anterior, la categoría de bienestar personal refleja que los individuos con artritis reumatoide del presente estudio son capaces de verificar la condición de su cuerpo y hacer adaptaciones en aquellos aspectos que más los afectan, a fin de lograr un estado de bienestar personal y de satisfacción consigo. De igual manera, se evidencia que los aspectos más difíciles de manejar cuando se sufre una enfermedad se relacionan con los ajustes en el estilo de vida, ya que implica desde iniciar el consumo de fármacos hasta sufrir modificaciones para la locomoción y movilidad como consecuencia de las dificultades y limitaciones en el movimiento. (Lugo, Salinas y Restrepo, 2008)

Respecto de la artritis reumatoide, es indispensable reconocer que puede provocar fatiga, inapetencia e, inclusive, sentimientos depresivos, así como alteraciones de sueño lo cual influye de forma importante en el bienestar de cada individuo y en sus actividades diarias, (Abasolo, Jover, León, Pérez, y Redondo, 2008). En relación con lo mencionado, Orem (1983) menciona que una patología no solo interviene con la fisiología, sino que afecta el funcionamiento humano, de ahí que las personas necesiten cambios en su modo de vivir. Por sus características, la enfermedad perturba el ámbito personal, laboral y económico y puede ocasionar disminución de la capacidad en los espacios mencionados y repercutir en la situación económica, personal, social y familiar. (Lugo, Salinas y Restrepo, 2008)

Otro elemento por considerar al evaluar la capacidad de agencia de autocuidado de una persona es la actividad y reposo (Orem, 1983): el estudio constató que el total de la población cuenta con alta capacidad como agente de autocuidado, conducta que influye positivamente en la salud, pues estos elementos constituyen requisitos básicos, propuestos por Orem (1983), considerados universales y elementales para los seres humanos (Orem, 1983). Para las personas con artritis reumatoide, la práctica de actividad física es una herramienta que puede ayudar a mejorar la flexibilidad y disminuir el dolor en las articulaciones (González, Novo, Reyes, y Toledano, 2007). Así mismo, el descanso, es clave para evitar un sobreuso de la articulación y proporciona un sueño placentero durante la noche, de manera que se reduce el dolor en el transcurso del día. (Príncipe y Ubilus, 2004)

En el penúltimo apartado, se discute la capacidad de agencia de autocuidado que poseen las personas con artritis reumatoide en relación con el consumo idóneo de alimentos, es decir, con una ingesta rica tanto en variedad como en cantidad, tanto de alimentos como de bebidas que generen bienestar y energía, con el fin de conservar y mantener la salud. 
Orem (1983) plantea que el consumo adecuado de nutrientes debe ser de acuerdo con la situación específica de salud de cada individuo, dato elemental para la población con artritis reumatoide, pues existe gran variedad de alimentos que contribuyen a contrarrestar los problemas articulares, de modo que reducen la inflamación y las molestias (Badillo, 2004). Al respecto, se encontró que el total de la población se ubica en el rango alta capacidad como agente de autocuidado, razón por la cual se debe trabajar intensamente como agentes de salud para mantener ese nivel, ya que si no se toma en cuenta podría generar complicaciones en la situación de salud, así como exacerbación de los síntomas o dificultad para la movilidad en estas personas, así lo muestran Abud, et al. (2005), quienes proponen que el mantener un índice de masa corporal elevado podría aumentar la progresión osteoarticular de la artritis reumatoide.

Finalmente, se discuten los resultados de la categoría de funcionamiento y desarrollo personal, definidas como actividades y modificaciones que realiza la persona para el mantenimiento de condiciones que apoyen los procesos vitales y promuevan el progreso humano.

Con la escala ASA se encontró que el total de la población se ubica en el rango alta capacidad como agentes de autocuidado en esta categoría, en la cual se abordan los temas relacionados con la promoción del desarrollo humano, la prevención de riesgos y el funcionamiento del cuerpo de acuerdo con la capacidad de la persona y sus limitaciones, lo cual puede contribuir a un mayor bienestar y desarrollo personal en el individuo.

Uno de los aspectos que se indagó el de los ajustes que debe realizar la persona, según las circunstancias, para cumplir con su autocuidado. Para entender lo anterior es necesario recalcar que la artritis reumatoide es una enfermedad inflamatoria crónica, en la cual se manifiesta un daño simétrico en el cartílago articular y zonas óseas proximales y una afección en las diversas articulaciones que van destruyéndose progresiva y lentamente (Farreras y Rozman, 2003; García, Quesada, 2004)). Por ello, es de suponer que el funcionamiento humano en términos de mecanismos fisiológicos y psicológicos debería estar afectado en los sujetos participantes del estudio. Sin embargo, el ASA muestra que, en la población del estudio ha logrado realizar el ajuste necesario para desempeñar su autocuidado.

Otro aspecto analizado fue la capacidad del individuo para cuidarse aún con las limitaciones presentes y modificaciones propias de la enfermedad; el fin de recabar tal información fue para que esta ayude a determinar si la persona puede valerse por sí o requiere de los sistemas de enfermería, especialmente, del sistema sustentador educativo, donde se promueve la capacidad de participación activa en el autocuidado y con ello se construyen sujetos capaces de satisfacer sus necesidades de autocuidado. (Orem, 1983)

La importancia de que el individuo cuente con la capacidad de participar en su autocuidado (Leiva y Rojas, 2009), radica en que él mismo determina sus necesidades y demandas, aunque en ocasiones el poder cumplir sus propios requisitos de autocuidado pueden verse imposibilitados. Por ello es relevante la participación activa de los sistemas de enfermería, establecidos por la teorizante, especialmente en las personas con artritis reumatoide, patología que disminuye tal capacidad. A partir de lo mencionado, se puede afirmar que la labor de Enfermería consiste en la satisfacción de las necesidades del individuo para contribuir con el desarrollo continuo del autocuidado, la recuperación y adaptación de los efectos de las patologías o lesiones sufridas, con la finalidad de mantener un estado de salud óptimo. (Marriner, Raile, 2007) 


\section{Revista Electrónica Enfermeria Actual en costa Rica}

\section{CONCLUSIONES}

- La población participante en el estudio mostró alta capacidad de agencia de autocuidado, en relación con los resultados obtenidos en la escala de Apreciación de la Agencia de Autocuidado (ASA).

- La escala ASA permitió a las investigadoras observar un panorama general de las necesidades de autocuidado correspondiente a la población participante, dato que sirve como base para posteriores intervenciones con un estudio similar.

- La escala ASA permite a la enfermera (o) determinar la capacidad de Agencia de Autocuidado, de forma oportuna y eficaz, además de que facilita el evaluar diferentes líneas de acción que satisfagan los requisitos de autocuidado, de modo que contribuya con la mejora del funcionamiento y desarrollo humano.

\section{REFERENCIAS}

Abasolo, L., Jover, J., León, L., Pérez, N. y Redondo, M. (2008). El dolor en los pacientes con artritis reumatoide: variables psicológicas relacionadas e intervención. Clínica y Salud, 19(3), s.p.

Abud, C., Díaz, E., Garza, M., Medrano, G., Orozco, J., Pacheco, C., Pineda, C., Pozos, J., Ramos, F., Robles, M. y Santana, E. (2005). Recomendaciones para el tratamiento médico de la artritis reumatoide. Revista de Investigación Clínica, 57(5), 735-755.

Álvarez, A., Anaya, J., Bonilla, L., Cadena, J., Cano, O., Correa, M., Gómez, M., Montoya, M., y Ortiz., D. (2002). Encuesta de conocimiento sobre artritis reumatoide: ¿Vale la pena educar?. Revista Colombiana de Reumatología, 9 (4), 262-269.

Anaya, J., Cadena, J., Tobón, S., y Vinaccia, S. (2004). Calidad de vida en las personas con artritis reumatoide a partir del cuestionario de calidad de vida en artritis. Revista Psicología y Salud, 14(1), s.p.

Araya, G., Mora, M., Ozols, A. y Villalobos, D. (2004). Perspectiva subjetiva de la calidad de vida del adulto mayor, diferencias ligadas al género y a la práctica de la actividad físico recreativa. Revista MHSalud, 1(1). Recuperado de http://www.una.ac.cr/mhsalud/documents/ARTICULOMONICAMORA_017.pdf

Arias, C. (2010). Entrevista sobre la población costarricense con artritis reumatoide. San Ramón, Costa Rica.

Badillo, R. (2004). Dieta y Enfermedades Reumáticas. Revista Salud UIS, 36,154-161.

Barrantes, R. (2002). Investigación: un camino al conocimiento un enfoque cualitativo y cuantitativo. San José, Costa Rica: Editorial Universidad Estatal a Distancia. 264.

Consejo de Organizaciones Internacionales de las Ciencias Médicas (CIOMS) (2002) Pautas éticas internacionales para la investigación biomédica en seres humanos. Programa Regional de Bioética OPS/OMS. Recuperado de http://www.paho.org/Spanish/BIO/CIOMS.pdf 


\section{Revista Electrónica Enfermería Actual en costa Rica}

Evers GC, Isenberg MA, Philipsen H, Senten M, Brouns G (1993). Validity testing of the Dutch translation of the appraisal of the self-care agency ASA-scale. Int J Nurs Stud, 30 (4), 331-42.

Farreras, P y Rozman,C. (2003). Medicina interna. (13ºd). Madrid, España: Editorial Harcourt, S.A. 1152.

Fernández, A. (2010). Efecto de una intervención educativa de enfermería en el fortalecimiento de la capacidad de agencia de autocuidado del adulto mayor hipertenso ambulatorio de Tunja-Boyacá, Colombia. Trabajo de Grado para optar por el título de Doctorado en Enfermería. Universidad Nacional de Colombia.

Fernández, A., Manrique, F. y Velandia, A. (2009). Análisis factorial de la Escala Valoración de Agencia de Autocuidado (ASA) en Colombia. Aquichan, 9(3), 222-235.

García, J. (2005). La bioética ante la enfermedad de Alzheimer. Editorial Desclée De Brouwer. España.

García, M. y Quesada, S. (2004). Artritis Reumatoide: fisiopatología y tratamiento. Centro Nacional de Información de Medicamentos. Instituto de Investigaciones Farmacéuticas. San José, Costa Rica: Editorial Universidad de Costa Rica. 76.

Gallegos, C. (1998). Validez y confiabilidades de la versión en español de la escala: Valoración de las Capacidades de Autocuidado. Desarrollo Cientifico Enfermería, 6(9), 260-266.

González, F., Novo, P. Reyes, G. y Toledano, G. (2007). Enfoque rehabilitador en la artritis reumatoide. Centro Nacional de Información de Ciencias Médicas. INFOMED Especialidades. Recuperado de http://www.sld.cu/sitios/reumatologia/temas.php?idv=23646

Johnson, M. Bulechek, G. Howard, B. McCloskey, J. Maas, M. Moordhead, S. y Swanson, E. (2007). Interrelaciones: NANDA, NOC y NIC (2 Edición). Barcelona: España. Editorial Elsevier. 693

Leiva, V y Rojas, L. (2009). Modelo conceptual de Imogene King y Dorothea Orem: Modelo autoinstruccional. San José, Costa Rica: Editorial Universidad de Costa Rica. 87.

Lugo, H., Salinas; F. y Restrepo, R. (2008). Rehabilitación en Salud. (2ºd). Medellín, Colombia: Editorial: Universidad de Antioquia. 902.

Marriner, A y Raile, M. (2007). Modelos y teorías en Enfermería. (6ed). España, Madrid. Editorial: Elsevier. 850.

Matud, M. (2008). Género y Salud. Colombia. Revista Suma Psicológica.15 (1), 75-94

Orem, D. (1983). Normas prácticas en enfermería. Traducido por Susan Allen. Madrid, España: Ediciones Pirámide, S.A. 294.

Principe, L. y Ubilus, W. (2004). Rehabilitación en artritis reumatoide. Diagnóstico, 43(4). Recuperado de http://www.fihudiagnostico.org.pe/revista/numeros/2004/jul-set04/158-161.html

Rigol, A. y Ugalde, M. (2001). Enfermería de salud mental y psiquiatría( $2^{\circ}$ Edición). España: Editorial Elsevier. 